\title{
A Study of the Yang-gyeong-gyu-il-ui (兩景揆日儀) in the Joseon Dynasty
}

\author{
Yong Sam Lee ${ }^{1}$, Sang Hyuk Kim ${ }^{2,3 \dagger}$, Byeong-Hee Mihn ${ }^{1,2,3}$ \\ ${ }^{1}$ Department of Astronomy and Space Science, Chungbuk National University, Cheongju 361-763, Korea \\ ${ }^{2}$ Korea Astronomy and Space Science Institute, Daejeon 305-348, Korea \\ ${ }^{3}$ Korea University of Science \& Technology, Daejeon 305-350, Korea
}

The Yang-gyeong-gyu-il-ui (兩景揆日儀) is a kind of elevation sundial using three wooden plates. Sang-hyeok Lee (李向赫, 1810 ?) and Byeong-cheol Nam (南秉哲, 1817 1863) gave descriptions of this sundial and explained how to use it in their Gyu-il-go (揆日考) and Ui-gi-jip-seol (儀器輯說), respectively. According to Gyu-il-go (揆日考) there are two horizontal plates and two vertical plates that have lines of season and time. Subseasonal (節候) lines are engraved between seasonal (節氣) lines, subdividing the interval into three equal lines of Cho-hu (初候, early subseason), Jung-hu (中候, mid subseason) and Mal-hu (末候, late subseason); there are 13 seasonal lines for a year, thus resulting in 37 subseasonal lines; also, there are 12 double-hour (時辰) lines for a day engraved on these plates. The only remaining artifact of Yang-gyeong-gyu-il-ui was made in 1849 (the $15^{\text {th }}$ year of Heon-jong) and is kept at the Korea University Museum. We have compared and analyzed Yanggyeong-gyu-il-ui and similar western sundials. Also, we have reviewed the scientific aspect of this artifact and built a replica. Yang-gyeong-gyu-il-ui is a new model enhanced from the miniaturization development in the early Joseon Dynasty and can be applied to the southern part of the tropic line through a structure change.

Keywords: Yang-gyeong-gyu-il-ui(兩景揆日儀), Hoeng-pyo-ip-pyo-il-gu(橫表立表日㫡), Sang-hyeok Lee, Byeong-cheol Nam, sundial, elevation dial

\section{INTRODUCTION}

While most of the sundials built in the late Joseon Dynasty were types of Angbu-ilgu (hemispherical sundial) or planar sundials (Kim et al. 2010), the Yang-gyeong$g y u-i l-u i$ is an elevation dial ${ }^{1}$. Jeon et al. (1984) performed a basic investigation of the Yang-gyeong-gyu-il-ui while they were doing research on Korea's scientific heritage. However, scientific analysis and verification have not yet been performed. Recently, Nha et al. (2013) and Nam \&

\footnotetext{
1) Yang-gyeong-gyu-il-ui was introduced by Byeong-cheol Nam when he published Ui-gi jip-seol, renaming the 'dial plate'(星表,) introduced in Gyu-il-go, which was published by Sang-hyeok Lee. When Nam \& Chen (2013) translated Ui-gi-jip-seol into Korean, they called it Yang-yeong-gyu-il-ui. In this study, the conventional name of Yang-
} gyeong-gyu-il-ui is used.
Chen (2013) have translated the Yang-gyeong-gyu-il-ui into Korean; those studies are referenced in this study.

Two articles having descriptions of the Yang-gyeong-gyuil-ui, Gyu-il-go (揆日考) and Ui-gi-jip-seol (儀器輯說) clarify that it was built based on the altitude of Hanyang (the old name of Seoul); it was an improvement of a conventional sundial achieved by applying a fork-type structure (' $\sqcup$ ') . We have compared the Yang-gyeong-gyu-il-ui and eastern and western sundials, etc., similar to the Yang-gyeong-gyu-il-ui, and have summarized the technical improvements. Also, we have reviewed the scientific aspect ${ }^{2}$ of this artifact and built a replica.

2) Measurement method, the engraving principle of 24 seasonal lines and time lines. (c) This is an Open Access article distributed under the terms of the Creative Commons Attribution Non-Commercial License (http:// creativecommons.org/licenses/by-nc/3.0/) which permits unrestricted non-commercial use, distribution, and reproduction in any medium, provided the original work is properly cited.
Received Dec 18, 2014 Revised Feb 23, 2015 Accepted Feb 26, 2015 †Corresponding Author

E-mail: astro91@kasi.re.kr, ORCID: 0000-0001-5297-928X

Tel: +82-42-865-2046, Fax: +82-42-861-5610 


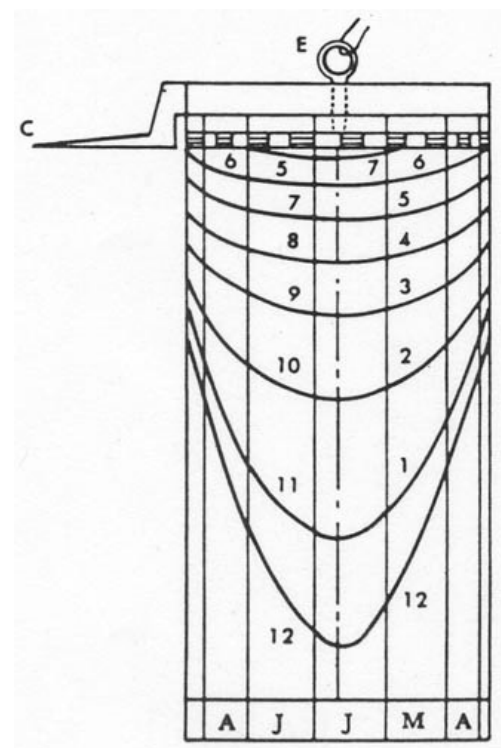

(a)

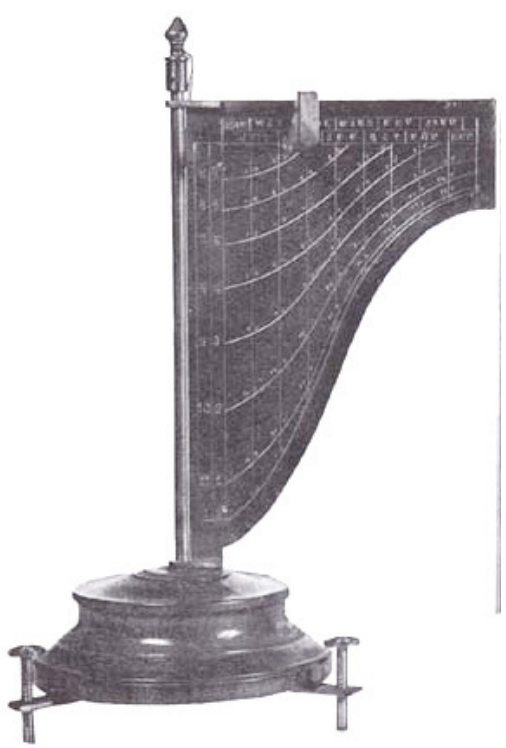

(b)

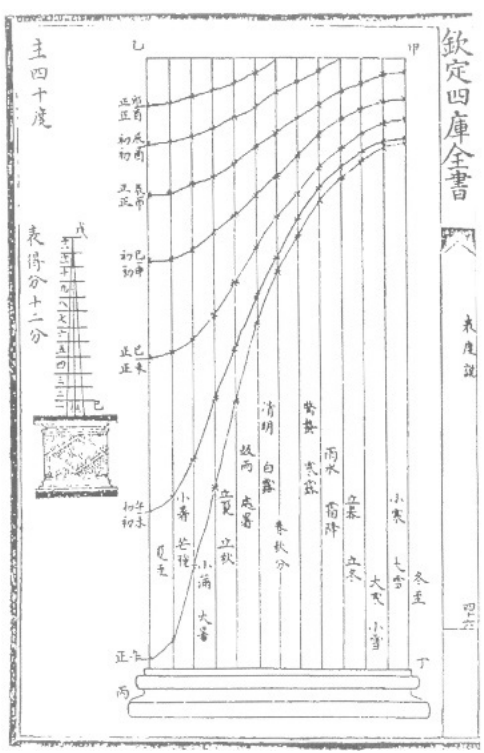

(c)

Fig. 1. Western Sundials.

(a) Shepherd's Sundial (Mayall \& Mayall 1994), (b) Elevation dial (18C) (Rohr 1970), (c) Biaodu Shuo (表度說) (Ji et al. 1781)

\section{ELEVATION DIAL}

\subsection{Shepherd's Sundial and Elevation dial of the West}

Elevation dials tell the time using the shadow of a gnomon according to the daily elevation of the Sun. In an elevation dial, there are several vertical lines to indicate seasons and it is possible to adjust the gnomon to set the dial to a specific time period of a year. In addition, there are curves crossing seasonal lines to indicate the time. Fig. 1(a) displays the Shepherd's sundial (Mayall \& Mayall 1994), which had been used in the Pyrenees up to recent times. Fig. 1 (b) shows an elevation dial (Rohr 1970); the hour lines of this dial represent a half of the cylinder shown in Fig. 1(a), when the cylinder is unfolded onto a plane.

Sabatino de Ursis (1575 1620), a missionary to China, introduced a western elevation dial in Biaodu Shuo (表度說). In Biaodu Shuo, there is a table showing the value of hour lines based on the altitudes of $30^{\circ}, 32^{\circ}$, and $40^{\circ}$. Following this table, de Ursis made a graph, which is shown in Fig. 1(c). The dial plates of these elevation dials are set up vertical to the ground; the gnomon is installed perpendicular to the dial plate.

\subsection{Hour and Seasonal Lines of Yang-gyeong-gyu-il-ui}

Sang-hyeok Lee (李向焃, 1810 ?) provided details of an elevation dial called a 'dial plate (㫡表)' in Gyu-il-go (揆 日考) ${ }^{4}$. This sundial has two horizontal plates (橫版 or 橫 表, hereafter 'the horizontal') and a vertical plate (立版 or 立表, hereafter 'the vertical'), on which the seasonal and hour lines are engraved; a sundial in which two plates are connected perpendicular to each other is called Hoeng-pyoip-pyo-il-gu (橫表立表日㫡). Lee augmented this sundial by adding a timeless plate (空表, hereafter 'the timeless') (Nha et al. 2013) to use it conveniently. Thus, the elevation dial in the Joseon dynasty is something of a newly developed threeplate dial made by adding the timeless to a conventional dial plate; the conventional dial plate came from China and had two plates, the horizontal and the vertical. Byeong-cheol Nam renamed the dial plate from Gyu-il-go, calling it Yanggyeong-gyu-il-ui in his work, Ui-gi-jip-seol. In this study, a dial plate with a timeless is called a Yang-gyeong-gyu-il-ui; a dial plate without a timeless is, for convenience's sake, called a Hoeng-pyo-ip-pyo-il-gu.

The conventional Hoeng-pyo-ip-pyo-il-gu has 13 seasonal lines and 12 double-hour lines. However, in Yang-gyeonggyu-il-ui, in Gyu-il-go, seasonal lines are drawn parallel to

4) The description of Gyu-il-go is based on the translated version of Nha et al. (2013) (Nha IS, Nam MH, Lee EH, Chen JJ, Gukyeok Yukiljae Chongseo2 (King Sejong the Great Memorial Society, Seoul, 2013), 323-347). 

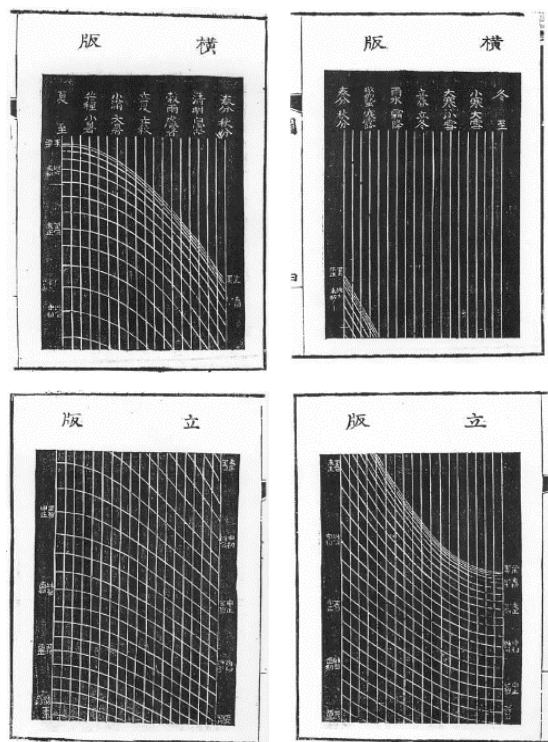

(a) 2- horizontal plate and 2-vertical plate

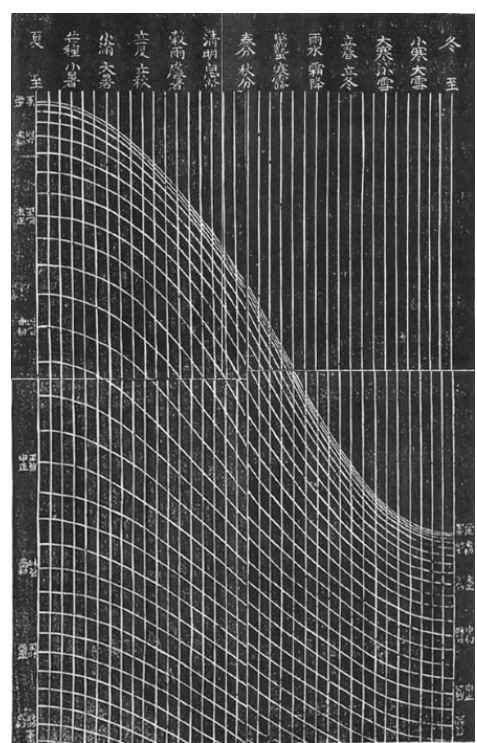

(b) 4 plates are combined.

Fig. 2. Yang-gyeong-gyu-il-ui ( $\left.{ }^{G} G y u-i l-g o 』\right)$.

the vertical direction and include 72 subseasonal lines, which are further subdivided into four equal parts by engraving subhour lines of curved shape at every true solar hour line.

The Yang-gyeong-gyu-il-ui of the Gyu-il-go consists of two horizontals and two verticals ${ }^{5}$ to be perpendicular to each two-plate made from copper or wood and a timeless, as shown in Fig.2 (a). The vertical is an erected plate and the horizontal is a horizontal plate; both have seasonal lines and hour lines engraved. The seasonal line is a straight line and the hour line is a curve.

As can be seen in Fig. 2 (b), placing two horizontals and two verticals in the order of 24-season, and then combining them gives the sundial hour lines and seasonal lines for a whole year. For 24 seasons, straight seasonal lines are engraved at fixed intervals; there are three lines subdividing each season equally. These are lines of 72 subseasons. However, in regard to the summer solstice and the winter solstice, the declination of the Sun is symmetric, and thus there are 13 seasonal lines and 37 subseasonal lines that are engraved; each subseasonal line indicates an interval of about 5 days.

The dial plate (時盤) of the Yang-gyeong-gyu-il-ui has parallel subseasonal lines engraved vertically and hour lines of S-shape engraved horizontally. At both ends of the hour lines, the time of 12 double-hours is marked and each interval is uniformly subdivided into 4 equal intervals of gak (刻; 15 minutes, quarter-of-hour); dividing a day into 96 gaks is a

5) According to ${ }^{\ulcorner} G y u$-il-go $o_{\lrcorner}$, figures of two horizontals and two verticals are shown, respectively; it is likely that one horizontal is connected to the other and one vertical is connected to the other one for usage. However, there is a possibility that these pieces are used separately for winter and summer seasons. typical time scale of the later Joseon Dynasty. In regard to instant, when the Sun passes the meridian, the hour angle of the Sun is symmetrical; thus, O-Cho(午初, 11:00)-Mi-Cho(未 初, 13:00), Sa-Jeong (巳正, 10:00)-Mi-Jeong(未正, 14:00), SaCho(巳初, 09:00)-Sin-Cho(申初, 15:00) etc., are marked with the same hour lines. On the other hand, Myo-Cho(卯初, 5:00)-SulCho(戊初, 19:00), Myo-Jeong(卯正, 6:00)-Yu-Jeong(西正, 18:00), Jin-Cho(辰初, 7:00)-Yu-Cho(西初, 17:00), and Jin-Jeong(辰 正, 8:00)-Sin-Jeong(申正, 16:00) hour lines are engraved on the vertical only; however, this could change according to the length of the horizontal. Investigating the dial plate shows that the hour line of noon starts at the top left, at the summer solstice $^{6}$, and goes to the bottom right in a curved shape.

In order to analyze the dial plate of the Yang-gyeong$g y u-i l-u i$, we have to investigate the variation of hour lines according to the changes in the altitude of the Sun. The shadow of the gnomon, set up vertically on a dial plate placed on the ground, shows a big difference between the winter solstice and the summer solstice. The shadow has become short at the summer solstice and has become long at the winter solstice.

If the vertical distances between the horizontal bottom line shown in Fig. 2(b) and the hour lines are assumed to be the length of the shadow, then it is longest at the summer solstice and shortest at the winter solstice. This is consistent with the behavior of a dial plate attached to a wall, from which the gnomon is installed horizontally. In a dial plate

6) If the latitude of the observer is lower than the Tropic of Capricorn, the time line cannot be drawn. 


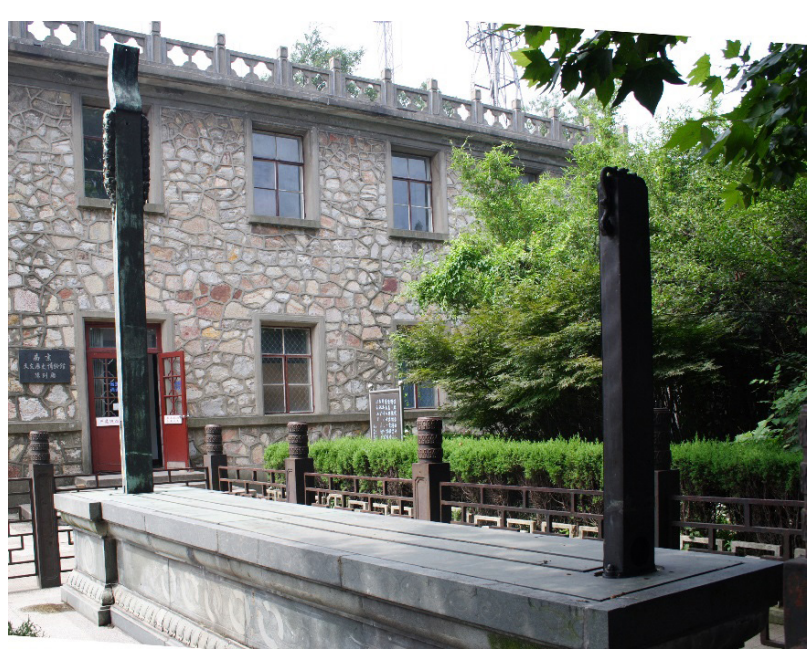

Fig. 3. Gyu-pyo (China Purple Mountain Observatory).

on a wall, the shadow of the gnomon is longest at noon and gets shorter as the altitude of the Sun drops. Similarly, if we let the vertical distances between the top horizontal line and the hour lines be the shadow lengths, they are shortest at the summer solstice and longest at the winter solstice. This is consistent with the behavior of a gnomon set up vertical to the ground on a dial plate placed horizontally. For a dial plate on the ground, the shadow length is shortest at noon and gets longer as the altitude of the sun drops.

It is described in Gyu-il-go that the Yang-gyeong-gyu-il$u i$ is used by combining the timeless, the horizontal, and the vertical. The timeless is used as a gnomon in the Yanggyeong-gyu-il-ui. Some of the western sundials mentioned above also use a vertical dial plate in a combined manner and have similar shaped dial plates. Meanwhile, the gnomon in the Purple Mountain Observatory of China has an 'additional gnomon (副表)' of shorter length on the north side, which enables a shortening of the length of the scales (圭) and gives a more circular-like inverse image of the Sun as the shadow gets shorter (Fig. 3). This addition of another gnomon (副表) is similar to the fork-type structure of the Yang-gyeong-gyu-il-ui.

\section{SHADOW OF THE HORIZONTAL AND THE VERTICAL}

If elevation dials are combined using a horizontal and a vertical, similar to the case of the Yang-gyeong-gyu-il-ui, there is a difference in the shapes of the hour lines. Fig. 4 shows the position of the shadow cast by the timeless on the horizontal or the vertical at the same times for the winter solstice and the summer solstice. In general, the shadow of a gnomon is formed on the dial plate, which is perpendicular to the gnomon; this shadow is called a direct shadow (直 影). Western elevation dials have longer shadows for the gnomon with the approach of the summer solstice. In order to complement this, a horizontal can be installed on the elevation dial, as shown in Fig. 4(a), making it possible to reduce the shadow length of the gnomon at the summer solstice. The shadow on a plane parallel to the direction of the gnomon is called a facing shadow (對影).

At the winter solstice, the altitude of the Sun is low, and the shadow of the timeless is formed on position $\mathrm{E}$ of the vertical (BC). On the other hand, at the summer solstice, the shadow of the timeless is formed on position $\mathrm{G}$ of the horizontal (CD). Without the horizontal, the shadow would be formed on position $\mathrm{F}$, which is on an extrapolation of the vertical. If we set the base of the shadow as B, the shadow length $s$ as a function of the altitude of the Sun, $\boldsymbol{a}$, can be expressed as in the following equation.

$$
\boldsymbol{s}=\left\{\begin{array}{cl}
\boldsymbol{l} \tan \boldsymbol{a} & \left(\boldsymbol{a} \leq \tan ^{-1} \frac{\boldsymbol{h}}{\boldsymbol{l}}\right) \\
\boldsymbol{l}+\boldsymbol{h}(1-\cot \boldsymbol{a}) & \left(\boldsymbol{a}>\tan ^{-1} \frac{\boldsymbol{h}}{\boldsymbol{l}}\right)
\end{array}\right.
$$

(from Point P)

Where, the length of the timeless or the horizontal is $l$; the length of the vertical is $\boldsymbol{h}$; and the elevation of the Sun is $\boldsymbol{a}$. If we let $\boldsymbol{h}=\boldsymbol{l}$, the above equation can be simplified into Equation (1).

$$
s=\left\{\begin{array}{cl}
\boldsymbol{l} \tan \boldsymbol{a} & \left(\boldsymbol{a} \leq \frac{\pi}{4}\right) \\
\boldsymbol{l}(2-\cot \boldsymbol{a}) & \left(\boldsymbol{a}>\frac{\pi}{4}\right)
\end{array} \quad\right. \text { (from Point P) }
$$

The shadow length of the timeless is $\boldsymbol{l} \tan \boldsymbol{a}_{\boldsymbol{W}}$ and $\boldsymbol{l}(2$ $\left.-\cot \boldsymbol{a}_{S S}\right)$ at the winter solstice and the summer solstice, respectively. The altitude of the sun is $\boldsymbol{a}_{S S}$ and $\boldsymbol{a}_{w S}$ at the summer solstice and the winter solstice, respectively.

In this case, the altitude of the Sun is obtained using spherical trigonometry, as follows:

$$
a=\sin ^{-1}(\sin \delta \sin \phi+\cos \delta \cos \phi \cos H)
$$

where $\boldsymbol{H}$ is the hour angle of the Sun; $\delta$ is the declination of the Sun; and $\phi$ is the latitude of the observer. The calculation method used in those days is also described in Gyu-il-go; the details can be found in Nha et al. (2013). In this study, modern calculation technology was used.

$\mathrm{AB}$ in Fig. 4 (a) and $\mathrm{AD}$ of Fig. 4 (b) are the timeless; Fig. 


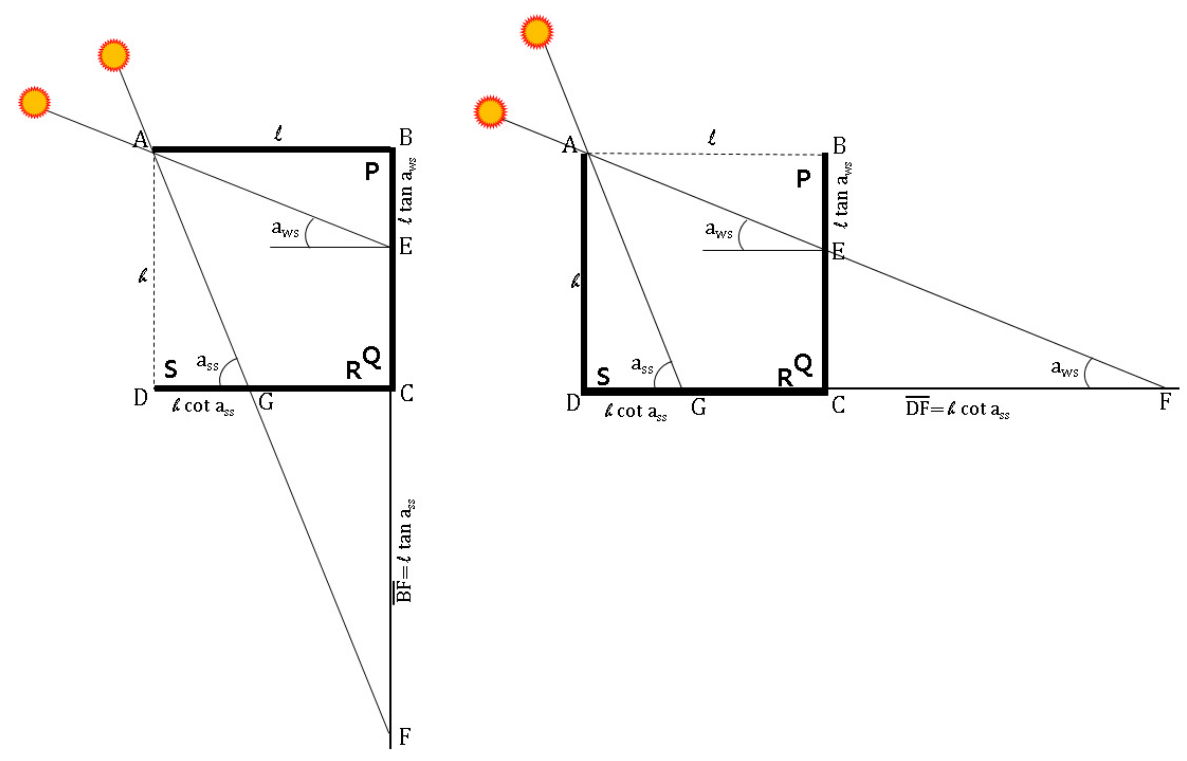

(a) the horizontal combined elevation dial

(b) Yang-gyeong-gyu-il-ui plate structure

Fig. 4. Shadow positions at winter solstice and summer solstice at the same time of day.

4 (b) shows the elevation dial augmenting the vertical. However, there are no changes in the positions or the shape of the hour lines on the horizontal, the vertical. The starting point of the shadow in Equation (1) is Point $\mathrm{P}$, which is consistent with Fig. 4 (b); if the shadow starts from Point $\mathrm{S}$ to be consistent with Fig. 4 (b), the shadow length can expressed as in the following equation,

$$
\boldsymbol{s}=\left\{\begin{array}{cc}
\boldsymbol{h} \cot \boldsymbol{a} & \left(\boldsymbol{a} \leq \tan ^{-1} \frac{\boldsymbol{h}}{\boldsymbol{l}}\right) \\
\boldsymbol{h}+\boldsymbol{l}(1-\tan \boldsymbol{a}) & \left(\boldsymbol{a}>\tan ^{-1} \frac{\boldsymbol{h}}{\boldsymbol{l}}\right)
\end{array}\right. \text { (from Point S) }
$$

According to Gyu-il-go, DG, DC+CE, and BE, shown in Fig. 4 (b), are defined as the directed shadow, the facing shadow, and the inverted shadow, respectively. In Equation (3), the two equations represent the direct shadow and the facing shadow, respectively.

If $\boldsymbol{h}=\boldsymbol{l}$ in Equation (3), the shadow length can be expressed as follows:

$$
s=\left\{\begin{array}{cl}
l \cot a & \left(a \leq \frac{\pi}{4}\right) \\
l(2-\tan a) & \left(a>\frac{\pi}{4}\right)
\end{array} \quad\right. \text { (from Point S) }
$$

Fig. 5 shows an elevation dial with a gnomon of $170 \mathrm{~mm}$ (Section 4) at a latitude of $37.5^{\circ}$ in Seoul with the dial plate of an elevation dial and with an augmented horizontal. In the figure, true solar time in 12 double-hours, the Joseonese time scale is shown; at every half double-hours, the line is plotted in black; at each gak, the line is plotted in grey. The seasons of Fig. 5 represents the winter solstice to the summer solstice one piece or vice versa, and is subdivided into 3 equal lines of Cho- $h u$ (初候, early subseason), Jung-hu (中候, mid subseason) and Mal-hu (末候, late subseason). Only 37 subseason lines are needed considering the superposition of lines among the total of 72 subseasonal lines.

The $\mathbf{P}, \mathbf{Q}, \mathbf{R}$, and $\mathbf{S}$ of Fig. 5 (b) represent the faces of the dial plates in Fig. 4 (a). In Fig. 5 (b), RS corresponds to the horizontal and corresponds to the vertical. Fig. 5 is plotted based on lengths of RS or PQ of $170 \mathrm{~mm}$. For lengths greater than $170 \mathrm{~mm}$, the shadow length shown in Fig. 5 (a) is longer than that shown in Fig. 5 (b); the ratio is the same as that of $\overline{\mathbf{C F}}$ to $\overline{\mathbf{C G}}$, shown in Fig. 4 (a). The difference between these ratios gradually increases as the latitude gets lower. Unlike the Yang-gyeong-gyu-il-ui, an elevation dial cannot be built in the tropics. Around the summer solstice in Fig. 5 (a), it is enable to measure the accurate time because the variation of the shadow length gets larger. While, in Fig. 5 (b), it is not easy to determine the time in detail because the interval between the hour lines is too narrow.

Typical sundials are installed to set the direction $O$ (午) to the true north. In other words, when the Sun crosses the meridian, the meridian direction of the sundial matches the meridian exactly, allowing us to tell the exact time. However, 


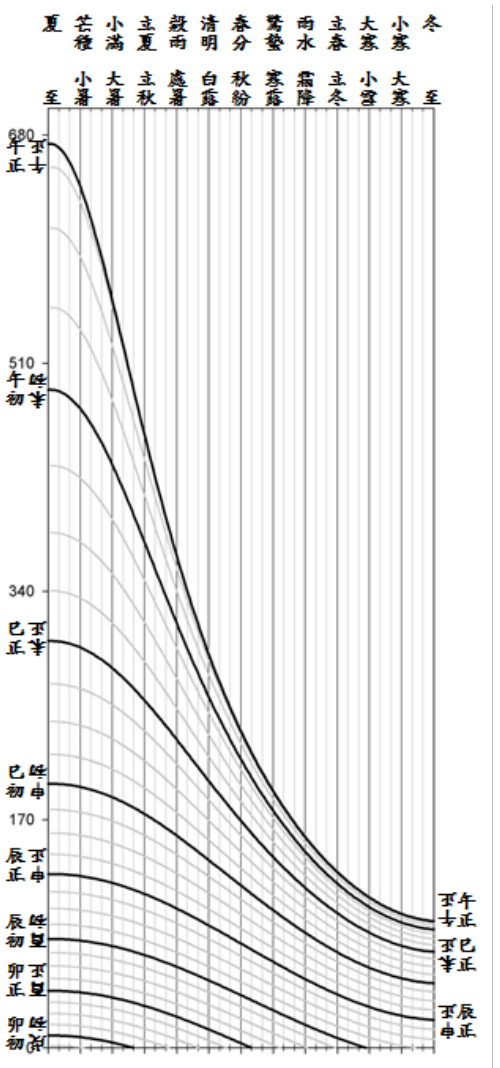

(a) Elevation dial

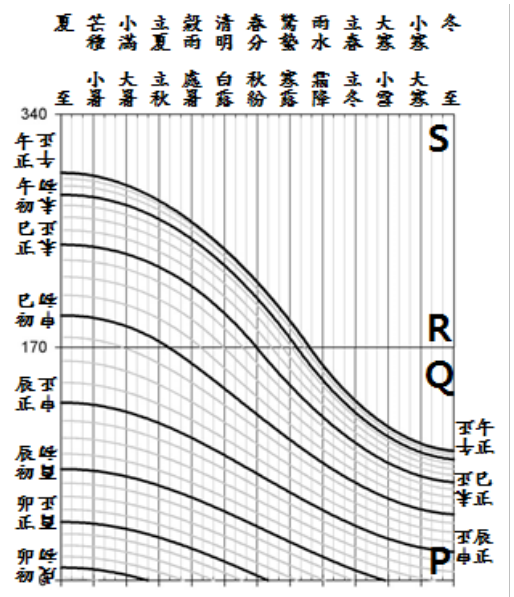

(b) The horizontals combined elevation dial

Fig. 5. Diagram of (a) Elevation sundial and (b) a horizontals-combined elevation dial with the time and seasonal lines cast by a timeless that is $170 \mathrm{~mm}$ long at a latitude of $37.5^{\circ}$ in Seoul.

a portable sundial requires us to set the direction of the sundial to the meridian; some sundials have a compass to enable this setting of the direction to approximately true north, based on magnetic north. In contrast, in the Yanggyeong-gyu-il-ui, we have only to make the dial plate, which is an assembly of the horizontal and the vertical, point to the Sun, regardless of the direction of meridian. Then, using the shadow of the top side of the timeless, when the end of the shadow crosses the subseasonal line, the hour lines are referenced and it is possible to read the time.

\section{THE STRUCTURE OF THE HOENG-PYO-IP-PYO- IL-GU}

Fig. 6 shows a Hoeng-pyo-ip-pyo-il-gu, the only remaining artifact of the Yang-gyeong-gyu-il-ui. This artifact was built in 1849 (the 15th year of Hyeon-jong) and is kept at the Korea University Museum. This artifact were measured $11.5 \mathrm{~cm}$ wide and $15.8 \mathrm{~cm}$ long by Jeon et al. (1984); Also, according to National Science Museum who had made the replica of it, the width, and the lengths of the horizontal and

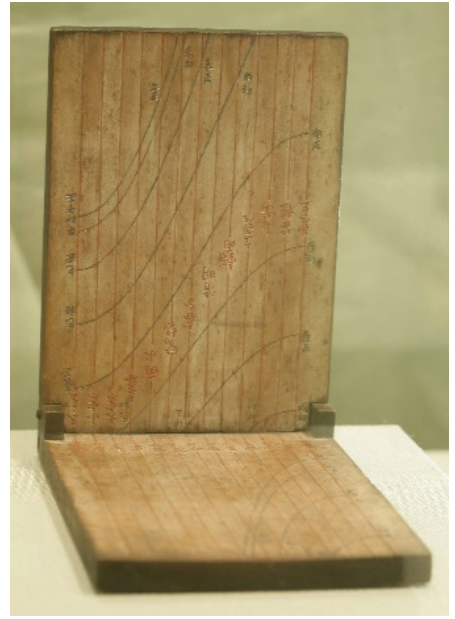

Fig. 6. Hoeng-pyo-ip-pyo-il-gu (Korea University Museum).

the vertical are suggested as $11.5 \mathrm{~cm}, 18.8 \mathrm{~cm}$, and $18.1 \mathrm{~cm}$, respectively ${ }^{7}$. This sundial has 13 seasonal lines and 12 hour lines engraved on it; thus, it is believed that this is a sample

7) It is necessary to do a precision measurement to determine these two values; in this study, the height of the Pyo (gnomon) is assumed to be $170 \mathrm{~mm}$ (reference for the replica dimensions: National Science Museum, 2013, Collection of the National Science Museum, Astronomy/Meteorology/Geography, p.82). 


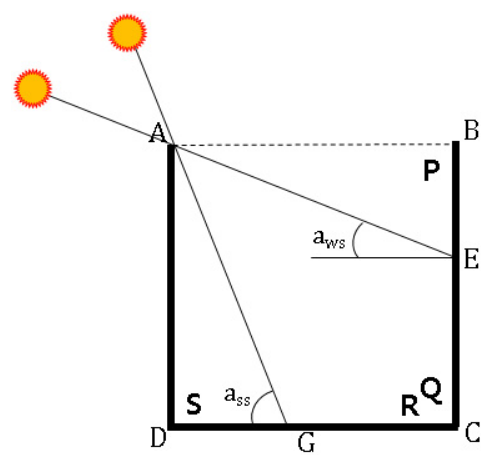

(a) Yang-gyeong-gyu-il-ui

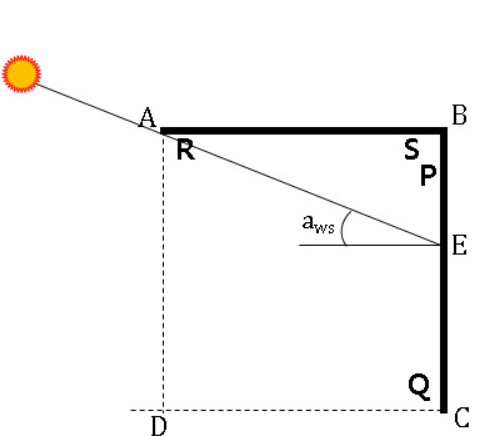

(b) Use as Ip-pyo (left) and Hoeng-pyo (right) in $\mathrm{HI}$

Fig. 7. Comparison of Yang-gyeong-gyu-il-ui to Hoeng-pyo-Ip-pyo-il-gu (HI dial).

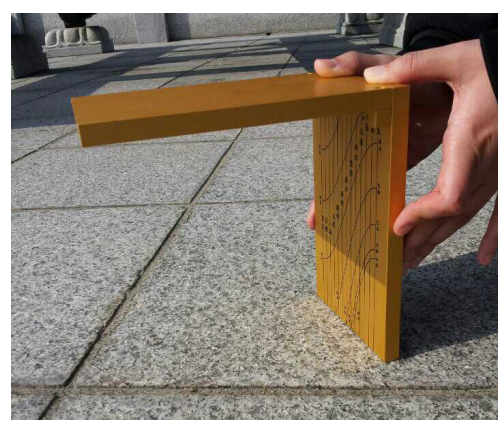

(a) Replica of HI dial

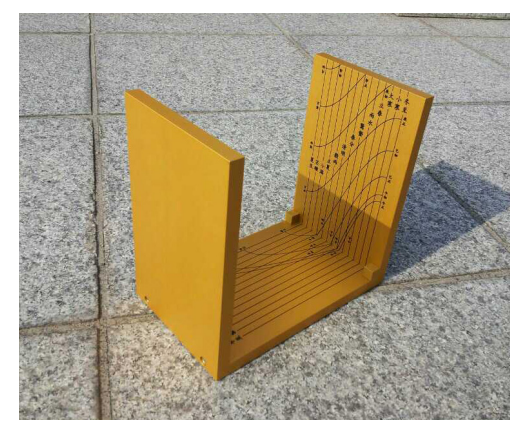

(b) Restoration of Yang-gyeong-gyu-il-ui

Fig. 8. Hoeng-pyo-Ip-pyo-il-gu (HI dial) and Yang-gyeong-gyu-il-ui.

of a Chinese dial plate as described in Gyu-il-go. Unlike the Yang-gyeong-gyu-il-ui, in this Hoeng-pyo-ip-pyo-il-gu, $\mathrm{P}$ and $\mathrm{S}$ coincide at the boundary of each plate, as depicted in Fig. 7 (b). Gyu-il-go explains this as the inverted shadow (倒影) of the horizontal.

The Yang-gyeong-gyu-il-ui can be represented as a ' $\sqcup$ ' shape. As can be seen in Fig. 7 (a), the Yang-gyeong-gyuil-ui can measure the shadow of the timeless regardless of the time if the horizontal is placed on the ground. In contrast, the Hoeng-pyo-ip-pyo-il-gu can measure the time if it is placed in a ' $L$ ' shape or in a ' $\neg$ ' shape. In the ' $\llcorner$ ' shape, the horizontal and the vertical are placed to have the vertical point at the Sun. At that moment, the hour lines of the horizontal are placed on the position of the inverted shadow. The disadvantage of the Hoeng-pyo-ip-pyo-il-gu is that it is difficult to maintain level of the horizontal when we measure the time when placing it in a ' $\neg$ ' shape as shown in Fig. 7 (b). However, the advantage of the Hoeng-pyo-ip-pyo$i l$-gu is that it is simple to build using just two plates.

Fig. 8 shows the newly built replicas of the Hoeng-pyoip-pyo-il-gu and the Yang-gyeong-gyu-il-ui, applying the latitude of the Korea Astronomy and Space Science Institute (KASI) $\left(36.4^{\circ}\right)$. On April $5^{\text {th }}, 2014$, we made a measurement of the time using the two types of sundials. Although there was a difficulty in leveling the Hoeng-pyo-ip-pyo-il-gu, there was no difference in the results. In particular, the replica of the Yang-gyeong-gyu-il-ui was built for the first time and it is expected that this replica will be utilized in education and exhibitions.

\section{CONCLUSIONS}

Based on figures from Gyu-il-go, authored by Sanghyeok Lee, we have investigated the hour lines of the Yanggyeong-gyu-il-ui and determined the usage. As described in the literature, this sundial was built applying the latitude of Seoul. While the time can be measured for known seasons, the intervals of the hour lines are subdivided into 15 minutes to improve the measurement of time.

Unlike a typical sundial, in which the direction of the gnomon is fixed, the gnomon of the western elevation dial is set up to point at the Sun to measure the time in a manner similar to that described in the Biaodu Shuo, from China. The Hoeng-pyo-ip-pyo-il-gu, which is the only remaining artifact of the elevation dial of the Joseon dynasty, has hour lines similar to those of an elevation dial; in this sundial, two plates abut perpendicular to each other to drastically reduce the size of sundial. Sang-hyeok Lee analyzed the theory behind the Hoeng-pyo-ip-pyo-il-gu and developed the Yang- 
gyeong-gyu-il-ui, which had a fork-type ('ப') structure to increase the stability of the sundial.

In this study, two technological features of the Yanggyeong-gyu-il-ui were found. First, the Yang-gyeong-gyu-il$u i$ is a compact sundial; unlike the western elevation dial (or those that have a large gnomon), the Yang-gyeong-gyu$i l-u i$ is an enhanced model resulting from miniaturization development in the early Joseon dynasty. Second, the Yanggyeong-gyu-il-ui is a new kind of elevation dial that can be used in south of the tropic through a modification of structure.

In addition, the technical improvement was confirmed through a comparison between the Yang-gyeong-gyu-il-ui and the western elevation dial. Furthermore, the principle of setting up the vertical in the Yang-gyeong-gyu-il-ui and the Hoeng-pyo-ip-pyo-il-gu was verified through geometrical analysis. Also, we have proposed a new replica model through astronomical calculation. Furthermore, we expect that this model will be used to appreciate the scientific and technological value of sundials in the late Joseon dynasty; this model will also be utilized in education and exhibitions.

\section{ACKNOWLEDGEMENTS}

This work was supported by a research grant from Chungbuk National University in 2012.

\section{REFERENCES}

Kim SH, Lee KW, Lee YS, A Study on the Sundials of the Kang Family of Jinju, JASS 27, 161-172 (2010). http://dx.doi. org/10.5140/JASS.2010.27.2.161

Jeon SW, Kim JS, Kim JH, Nha IS, Nam CW, et al., Research Report on the Science and Cultural Heritage of Korea, KJHS 6, 85-88 (1984).

Ji Y, Lu XX, Sun SY, Lu FC, Biaodu Shuo (表度說) (Wenyuange edition of Siku Quanshu (文淵閣 四庫全書), Beijing, 1781), 42-48.

Mayall RN, Mayall MW, Sundials: Their Construction and Use (Sky Publishing Corp., Cambridge, 1994), 165.

Nam MH, Chen JJ, Gukyeok Yukiljae Chongseo 1 translated in Korean (King Sejong the Great Memorial Society, Seoul, 2013), 317-321.

Nha IS, Nam MH, Lee EH, Chen JJ, Gukyeok Yukiljae Chongseo 2 translated in Korean (King Sejong the Great Memorial Society, Seoul, 2013), 323-347.

Rohr RRJ, Sundials: History, Theory, and Practice (University of Toronto Press, Toronto, 1970), 71. 